\title{
Flexural behaviour of Carbon and Glass fiber composite laminates reinforced with Nylon 6,6 electrospun nanofibers
}

\author{
R. Palazzetti* \\ Department of Design, Manufacture \& Engineering Management \\ (DMEM), University of Strathclyde, Glasgow (UK)
}

\begin{abstract}
Nylon 6,6 nanofibers manufactured by means of electrospinning have been used to interleave Mode II fracture mechanics glass and carbon unidirectional (UD) fiber composite specimens. The aim of this work was to study the effect of the nanofibers materials in their ability to reinforce the interleave. Experimental testing was carried out on specimens with a nanofibrous mat interleaved into a delaminated interface. Specimens of 10, 16 and 18 layers were manufactured and tested. Results demonstrated that the effect of nanofibers was different between the two materials and that the fibers material plays an important role in the reinforcement mechanism of the nanofibers.
\end{abstract}

Keywords: Nanofibers, Electrospinning, Mechanical Testing, Composite laminate.

\section{INTRODUCTION}

The attempt to strength laminate interfaces by interleaving nanofibrous mats was an approach first developed by Dzenis and Reneker in 1999 [1]. In the last decade, research on composite laminates interleaved with nanomats has boomed due to the fact that the nanointerleave is able to strengthen the laminate's delamination resistance without affecting out of plane properties such as flexural stiffness, thickness or weight [2-6].

However, despite interleaving composite laminates with nanofibers has a strong potential to improve delamination resistance [7], the nanointerleave requires careful design consideration, as doing so under the wrong conditions may lead to significant negative effects [8-10].

The present paper considers the application of Nylon 6,6 nanofibers. Few published works to date have dealt with the use of such a reinforce and two of the most important ones are addressed to Shivakumar [11] and Akangah [12]. The first performed experiments proving that Nylon nanofibers interleaved into

*roberto.palazzetti@strath.ac.uk 
uni-directional (UD) Carbon Fiber Reinforced Plastic (CFRP) were able to enhance dynamic properties, impact damage resistance, fracture toughness, and delamination onset life. The second investigated the same raw material but focused on the impact behaviour. Sixteen-ply, quasi-isotropic composite laminates were manufactured and impacted to assess the improvement in impact resistance given by the nanointerleave.

Despite the large amount of research dealing with the toughening effect of nanofibers applied into composite interfaces, the effective reinforce mechanism is still a partially uncovered topic. In previous work of Palazzetti [13], a presentation is given on the effect of nanointerleave's geometrical features (such as thickness, fiber diameter and fiber orientation) on woven CFRP Mode I and Mode II loaded specimens.

Nanofiber's toughening mechanism has been investigated in [14, 15], focusing on the resin interaction with the nanomat, the presence of nanofibers increases the damping of the laminate by friction with the undamaged resin, enabling crack-bridging when the matrix breaks. Since a significant nanofibers-bridging has been detected it is worth investigating how much the microfibers-nanofibers interaction weights on the nanomat's reinforce effect. For this purpose, the present work is focused on the effect of the number of layers and the fibers material selection on the nanomat's reinforce mechanism.

End Notched Flexure (ENF) specimens have been manufactured using UD glass and carbon prepreg; two sets of experiments were implemented to study the influence of $(i)$ the number of laminate's layers and (ii) the fibers material, on the effect of the nanoreinforce.

\section{Materials And Methods}

\subsection{NANOFIBERS}

Nanofibers were manufactured by means of electrospinning of Nylon 6,6 Zytel E53 NC010 (Table 1) kindly provided by DuPont company.

\begin{tabular}{rc}
\hline Property & Value \\
Nominal Strain at Break & $>50 \%$ \\
Yield Strain & $4.4 \%$ \\
Tensile Modulus & $3000[\mathrm{MPa}]$ \\
Notched Charpy Impact Strength & $7\left[\mathrm{~kJ} / \mathrm{m}^{2}\right]$ \\
\hline
\end{tabular}

Table 1: Nylon 6,6 properties (Source: DuPont)

The polymer was dissolved in a solution made of Formic Acid and Chloroform (50:50 v/v) purchased from Sigma Aldrich, used without further purification. Electrospun non-woven mats were fabricated by using a SPINBOW S.r.l. electrospinning semi-automatic machine ${ }^{1}$, composed of a high voltage power supply, a double syringe pump, two chambers containing the polymeric solution (each one equipped with four stainless- steel blunt-ended needles and connected with the power supply electrode) and a grounded plane collector positioned $10 \mathrm{~cm}$ away from the tip of the needles. The electrospinning process was carried out

\footnotetext{
${ }^{1}$ Via dell'Artigiano 8/6, 40016 San Giorgio di Piano, Italy. info@spinbow.it
} 
under the following conditions: applied voltage $22-26 \mathrm{kV}$, feed rate $0.3 \mathrm{~mL} / \mathrm{h}$ per nozzle, at room temperature and relative humidity $\mathrm{RH}=40-50 \%$. A Nanofibrous mat is presented in Figure 1.

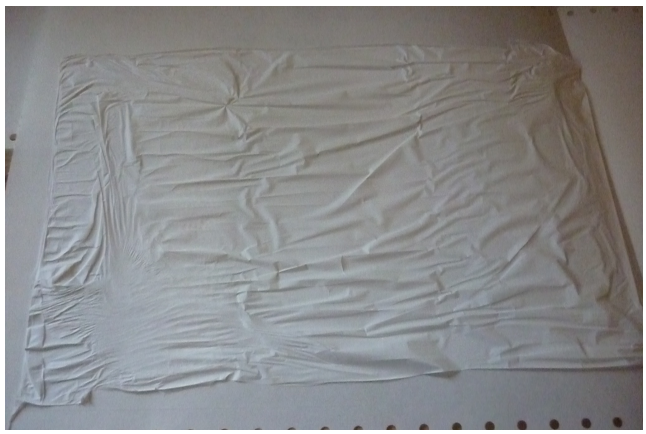

(a) Nylon 6,6 nanofibrous mat

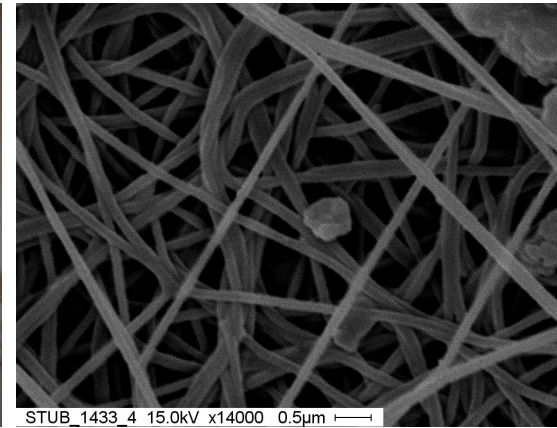

(b) SEM image of the nanomat

Figure 1: Images of the Nylon 6,6 nanofibers

Electrospun mats were kept under vacuum at room temperature overnight to remove residual solvents before the lay-up inside laminates. Thermal properties of Nylon 6,6 electrospun mat were investigated by means of differential scanning calorimetry (DSC) using a TA Instruments Q100 DSC equipped with a Liquid Nitrogen Cooling System (LNCS) low-temperature accessory. Nanofibers are characterized by a high-melting crystal phase (peak temperature $T_{\text {melting }}=$ $262^{\circ} \mathrm{C}$, and $\Delta H_{\text {melting }}=65 \mathrm{~J} / \mathrm{g}$ ); prepreg curing treatment, carried out at $130^{\circ} \mathrm{C}$, is then below the Nylon 6,6 melting temperature and does not cause any modification at mat shape or fiber morphology. The Nylon 6,6 nanofbers electrospun for the experiments here presented, have been already used in other work of the author $([3,13,14,16])$, and proved to maintain their integrity when subjected to the thermal and pressure stresses applied into the autoclave. Furthermore $[13,14,16]$ already proved that Nylon 6,6 nanofibers get completely wet by the resin, during the cure in autoclave. Furthermore, the curing process is monitored by a sacrificial item placed into the autoclave, together with the specimens, with a thermocouple inserted in it to check the real temperature of the resin while it cures, to ensure that the nanofibers do not melt.

Nanofibers properties are very difficult to determine, due to the tiny dimensions of the fibers, and in literature few works have been presented on the topic. It is known that mechanical properties of the nanofibers strongly depend on nanofiber's diameter and process's condition [17]; in particular, the main feature governing nanofiber's mechanical strength and stiffness is the macromolecular alignment: in this work $100 \mathrm{~nm}$ nanofibers have been manufactured, which ensures a significant molecular orientation's grade.

\subsection{COMPOSITE SPECIMENS}

ENF beam-like specimens were manufactured by using epoxy matrix/glass and carbon fibers UD prepregs with fiber aligned parallel to the length of the beams. The epoxy matrix used was a tetrafunctional epoxy monomer, tetraglycidylether of 4,40 diaminodiphenil methane (TGDDM), and a difunctional epoxy monomer, 
bisphenol A diglycidyl ether (DGEBA), while 4,40 diaminodiphenylsulfone (DDS) was used as hardener. The weight ratio of the three components was 100:19:31 (TGDDM:DGEBA:DDS). All reactants were supplied by Sigma Aldrich and used as received. The carbon fibers (UNIC CUT 300/10 HMU659 10 HM) and glass fibers (Ref. 1017, glass fiber EC9 5x136 tex) have been purchased from Dalla Betta Group Srl and Angeloni Srl respectively.

Carbon fiber specimens were manufactured with 18 layers. Glass fibers specimens were manufactured in 2 different configurations: 10, and 18 layers. The results presented in [16] have been also considered, in particular those of the ENF tests on 16 layers, glass fibers specimens. In Table 2 manufactured specimens are summarized.

\begin{tabular}{cccc}
\hline Material & Width $(\mathbf{m m})$ & $\mathbf{N}^{\circ}$ of layers & Thickness $(\mathbf{m m})$ \\
Glass & 20 & $10-16^{*}-18$ & $2.35-4.20^{*}-4.97$ \\
Carbon & 20 & 18 & 4.23 \\
\hline
\end{tabular}

* Results taken from [16]

Table 2: Laminate's configurations

Virgin and nanomodified specimens were manufactured, and the thickness of the latter did not register an increase due to the presence of nanofibers with respect to the virgin specimens. Five specimens of each configuration have been manufactured, and the results are given in terms of average and standard deviation. In order to reduce experimental errors, all the specimens have been manufactured together in one process. Furthermore, each configuration has been manufactured in one big lamina, from which the samples have been cut out using a rotating diamond saw.

Specimens have been cured in autoclave at University of Bologna Forli's laboratory, according to the supplier's specifications.

Surface density of the carbon fibers, glass fiber and nanomats are 300, 430 and $9 \mathrm{~g} / \mathrm{m}^{2}$ respectively, thus the nanofiber content was equal to $0.19 \%, 0.12 \%$ and $0.10 \%$ for the 10, 16 and 18 layers glass specimen respectively, and equal to $0.15 \%$ for carbon specimens. Weight content of nanofibers can thus be considered negligible.

Since a proper Mode II testing methodology has not been standardized yet, experiments are carried out taking inspiration by the International Standard ASTM D 7264 [18], as shown in Figure 2(a).

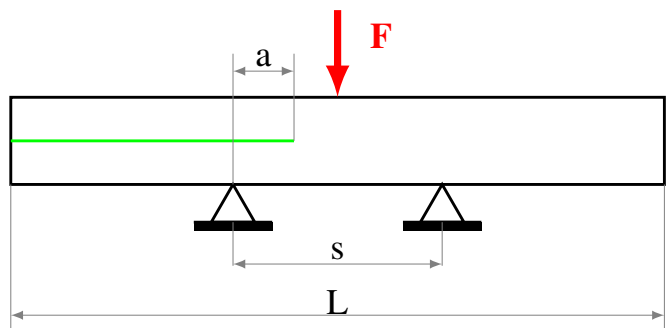

(a) ENF sample. Figure not to-scale

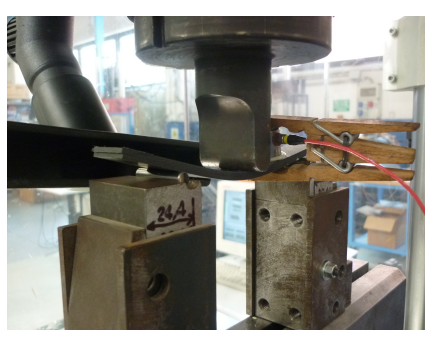

(b) ENF Test

Figure 2: ENF tests 
Specimen's length $(L)$ was $150 \mathrm{~mm}$, force $(F)$ was applied in the middle of the $60 \mathrm{~mm}$ span $(s)$ and the crack length $(a)$ entering into the support span was $15 \mathrm{~mm}$. Delamination was created by laying down a $15 \mu \mathrm{m}$ Teflon sheet during the lay-up on one side of the specimens: it avoids the resin bonding the layers and creates the crack tip the delamination will propagate from. The size of the Teflon was as wide as the width of the specimens

Despite the [18] recommends that the support-to-span ratio ranges from 16 to 60 , here lower ratios have been used: ratios of 25, 14 and 12 have been adopted for the 10, 16 and 18 layers specimens respectively. This is due to the fact that Mode II tests aim to load the delaminated interface in shear mode, and thus the effect of the shear is encouraged to be as high as possible (which is something to avoid when testing sample to identify flexural properties).

Nanomodified specimens are interleaved with a layer of Nylon 6,6 nanofibers applied in the delaminated interface during the lay-up, as presented in [13].

\section{EXPERIMENTS}

As recommended in [18], experiments were carried out under displacement controlled conditions at a constant cross-head rate of $1 \mathrm{~mm} / \mathrm{min}$, in a servohydraulic universal testing machine Instron 8033, with a $1 k N$ load cell applied on the loader to record the force. Load and cross-head displacement were recorded 10 times per second during the test. Support rollers were $8 \mathrm{~mm}$ diameter steel pins, Load was applied to the specimens via a $9 \mathrm{~mm}$ radius penetrator as shown in Figure 2(b).

Direct beam theory was used to calculate the critical energy release rate for Mode II $\left(G_{I I C}\right)$ using the [19]:

$$
G_{I I C}=\frac{9 \cdot a_{c}^{2} \cdot P_{c} \cdot \delta_{c}}{2 \cdot B \cdot\left(2 \cdot L^{3}+3 \cdot a_{c}^{3}\right)}
$$

where $P_{c}$ is the maximum load, $\delta_{c}$ is the loader displacement at the maximum load, $L, b$ and $h$ are the specimen's length, width and thickness respectively. Crack propagation was measured by visual inspection using a high-resolution camera pointing the crack tip on the outside of the specimens.

Stress and strain of the outer surface are determined throughout the tests following the [18]:

$$
\sigma=\frac{3 \cdot P \cdot L}{2 \cdot b \cdot h^{2}} \quad \epsilon=\frac{6 \cdot \delta h}{L^{2}}
$$

Curves are plotted in Figure 3 and 4.

The presence of delamination makes the specimen not symmetric with respect to the loader axis, and unbalance the stress field. Consequently the stressstrain curved calculated with the (2) do not represent the real stress state of the specimens, as it happens for all the ENF experiments. In particular the numbers plotted on the charts are slightly lower than the real values: the experiments underestimate how an intact sample would respond to the load. The point is that the focus here is not on the real absolute values of stresses and strains, but on the effect that the nanofibers has when interleaved in a sample, and then on the differences between a nanomodified and a non-nanomodified specimen. For this reason the $\sigma-\epsilon$ are used to compare two situations, but they are not meant 
to be taken as absolute values.

Figure 3 presents the Stress vs. Strain curves for the glass fibers specimens: Figures 3(a), 3(b) and 3(c) report the experimental curves for the 18, 16 and 10 layers' specimens respectively. To make the charts clearer and more readable, only the most representative curve of each case has been reported. Nevertheless, the results presented in the charts of Figure 5 will report the error bar.

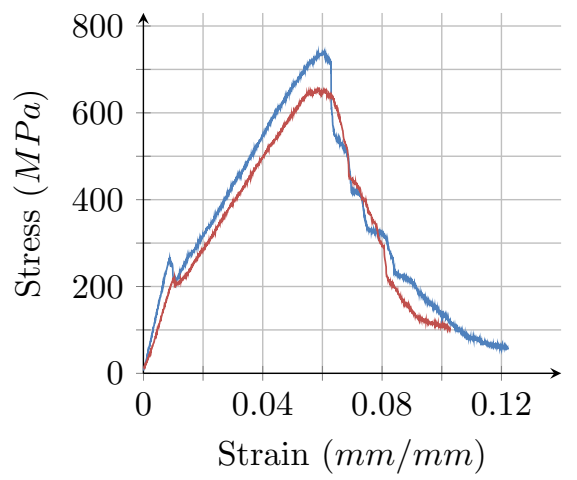

(a) 18 layers specimens

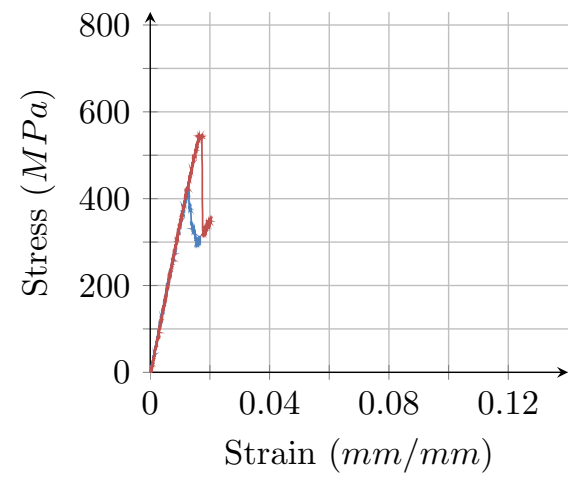

(b) 16 layers specimens

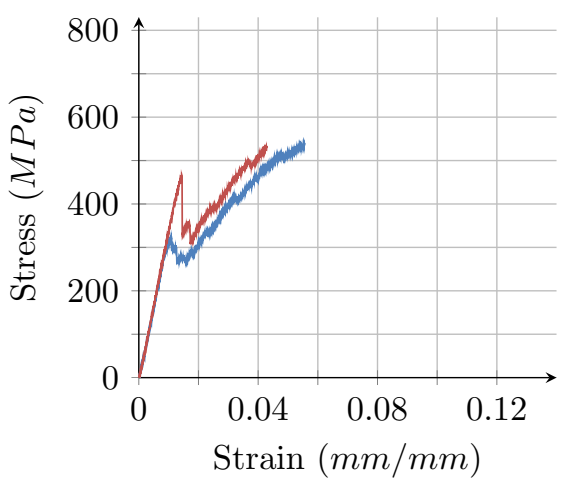

(c) 10 layers specimens

Figure 3: Stress vs. Strain curves of Glass specimen. Blue [Red] lines refer to virgin [nanomodified] specimens

It is worth highlighting that the nanofibers do not influence the stiffness of the specimens, the initial linear part of the curves of virgin and nanomodified specimens perfectly overlaps. However, the effect of interleave on the load capacity is clearly visible as soon as the crack begins to propagate.

Figure 4 reports the Stress vs. Strain relations for Glass (4(a)) and Carbon (4(b)) fibers with 18 layers' specimens showing different effect of the nanofibers, which will be discussed in $\S 4$.

\section{Discussion}

Maximum stress $\left(\sigma_{\max }\right)$ and critical energy release rate $\left(G_{I I C}\right)$ have been used as mechanical parameters to compare the two tested configurations and to measure 


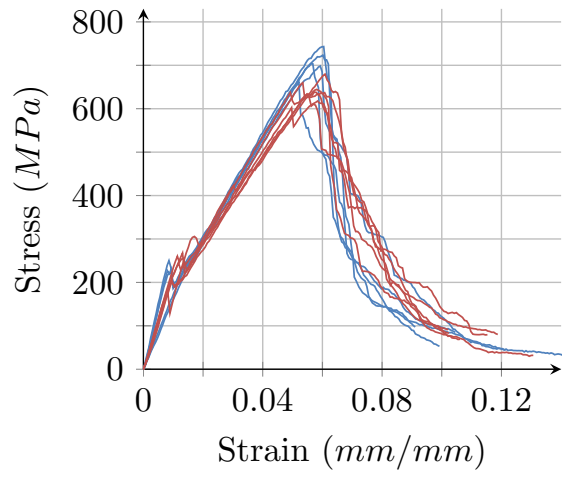

(a) Glass fiber specimens

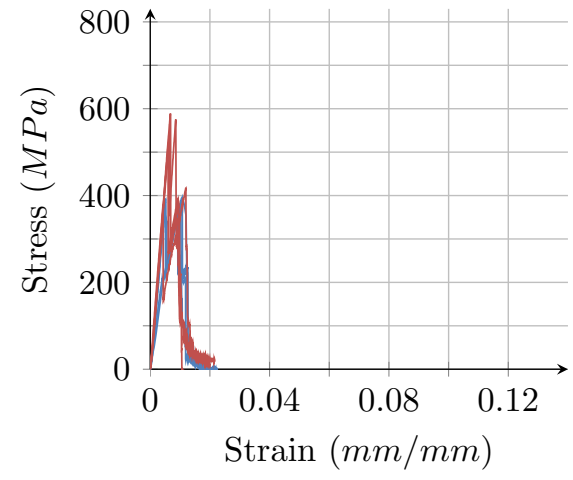

(b) Carbon fiber specimens

Figure 4: Stress vs. Strain curves of 18 layers specimens. Blue [Red] lines refer to virgin [nanomodified] 18 layers specimens

the efficacy of the nanointerleave. The ratios between the $\sigma_{\max }$ and $G_{I I C}$ of nanomodified specimens with those of virgin are presented to highlight the effect of the nanointerleave.

Figure 5 presents the results of Glass fiber specimens.

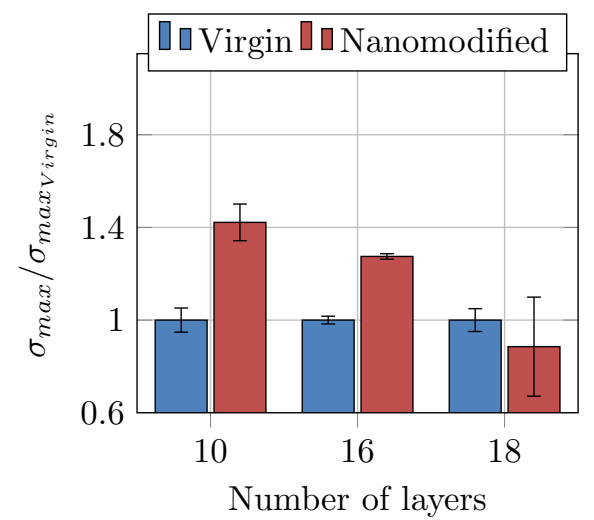

(a) $\sigma_{\max } / \sigma_{\max } \operatorname{mirgin}$

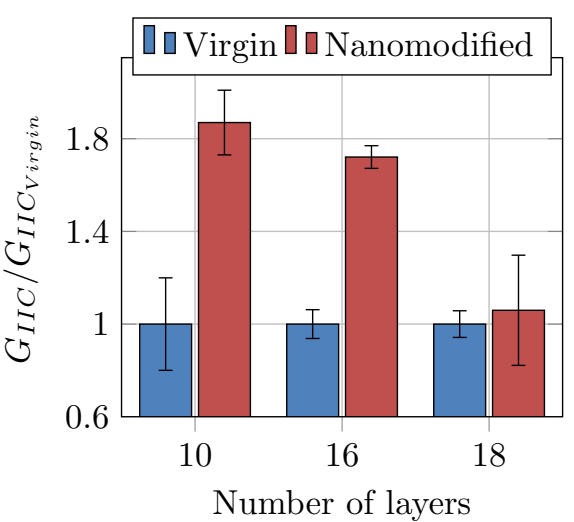

(b) $G_{I I C} / G_{I I C_{\text {Virgin }}}$

Figure 5: Experiments results for Glass specimens

Results, as expected, demonstrate a decreasing effect of the nanomodification as the number of layers increases due to the fact the shear stress, proportional to the section area, decreases as the thickness increases. As the number of layer increases, the shear highest value, located at the crack tip and proportional to the force-to-surface ratio, decreases, and at the same time the nanoreinforce effect is also reducing. Experiments demonstrated that the nanointerleave still has significant effect into 16 layers specimens, which is remarkable considering the small amount of nanofibers compared with the mass of resin and fibers present in the specimens. 
Both Figure 5(a) and Figure 5(b) show an unexpected high scatter for 18 layers nanomodified specimens, however, the purpose of this paper is to evaluate the influence the material and the number of layers have on the effectiveness of the nanoreinforce and despite the high scatter of the thickest configuration, all nanomodified specimens register higher parameters than the virgin ones.

Figure 6 presents the $\sigma_{\max } / \sigma_{\text {maxVirgin }}$ and $G_{I I C} / G_{I I C V i r i g n}$ ratios comparing Glass and Carbon fibers, 18 layers specimens. Results are normalized respect to the virgin samples' results to enhance the effect of nanointerleave.

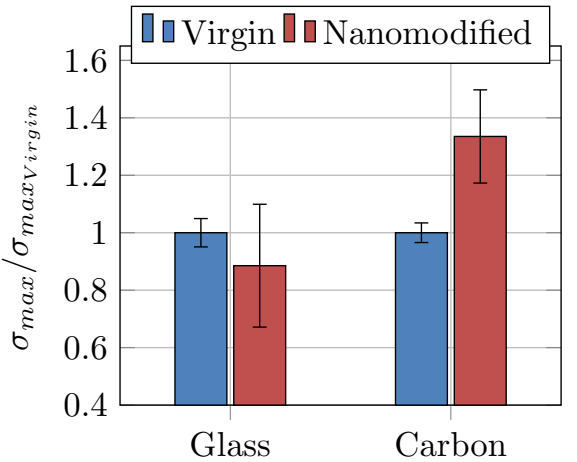

(a) $\sigma_{\max } / \sigma_{\max \operatorname{Virgin}}$

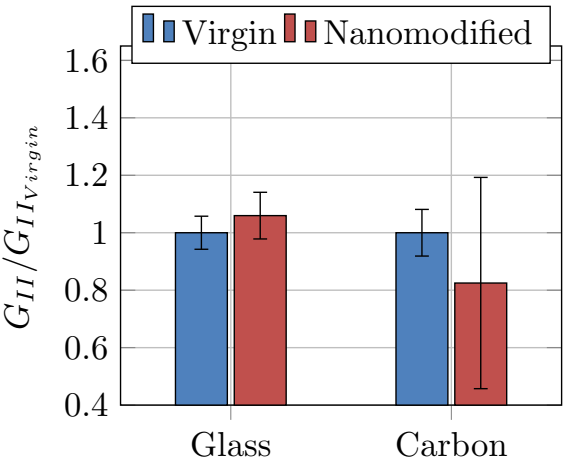

(b) $G_{I I C} / G_{I I C_{V i r g i n}}$

Figure 6: Experiments results for 18 layers specimens

The charts in Figure 6 do not present a clear picture of the material's effect on the behaviour of the nanomodified specimens, due to the high scatter of the nanomodified specimens.

Despite the high error bar, Figure 6(a) clearly shows that the Nylon 6,6 nanofibers in Carbon fiber specimens are able to increase the maximum load the specimen can carry. On the other hand no significant conclusion can be drawn from Figure $6(\mathrm{~b})$.

The reason behind the high scatter recorded for the 18 layers specimens is due to the short support span adopted for the experiments. In order to increase the shear component, the support span was kept short, and it induced experimental errors, due to misalignment. The shorter the span, the higher the influence of non correct alignment of the specimen is, and thus the higher scatter registered for the thickest specimens.

In a previous work of the author [14], the interaction of nanofibers with resin was investigated, demonstrating that the friction between them is responsible for increasing the damping of the material, and that the nanofiber-bridging after crack propagation is able to strengthen the interface. Results presented here make a step forward on the knowledge of the behaviour of nanofibers interleaved into laminates, presenting a more complex reinforce mechanism, also taking into account the amount of microfibers and the material selection.

The key aspect of the nanofibers' role is the strain the specimens are subjected to. Figure 4 shows that the elastic field of the glass fibers reaches $0.06 \mathrm{~mm} / \mathrm{mm}$, while that of the carbon is lower than $0.01 \mathrm{~mm} / \mathrm{mm}$. Nanofibers are put in tension as soon as the test begins and the laminate is loaded. Until the nanofibers are intact, they are capable to carry on the load, increasing the maximum stress 
that the specimen would carries on without them (like in the Carbon specimens, see Figure 4(b)). When nanofibers are applied into a more compliant material, like the glass fiber, they are subjected to a higher strain, which breaks them before reaching the load peak (see Figure 4(a)).

It could be argued that the specimens do not reach the $4.4 \%$ strain indicated in table 1 to break the fibers; on the other hand, instead, the fibers strain much more than their nominal strain, in particular on the delaminated part of the interface. The strain used to draw the curves depends on the displacement of the loader and are calculated by using the second formula presented in (2), considering an undamaged item. In correspondence of the delamination, instead, the specimens are divided in two parts, which slides one another much more than the $4.4 \%$ that would break the nanofibers.

\section{CONClusions}

As reported in current literature, a nanomat interleaved into a composite interface leads to a significant nanofibers-bridging, demonstrating nanofibersmicrofibers interaction. The focus of this work is to demonstrate how the interaction between nanofibers and microfiber is significant in the nanomat's reinforce mechanism. In particular, the influence of the material selection and of the number of layers on the effectiveness of the nanoreinforce has been investigated. For this purpose an experimental testing was carried out to investigate the behaviour of thick composite laminate specimens interleaved with Nylon 6,6 nanofibers under Mode II fracture mechanics load. Interleaving nanoreinforce in glass fiber specimens proved to be effective in laminates made of 10 and 16 layers, while for thicker specimens the effect of the reinforce is almost negligible, despite the high scatter of the 18 layers glass fiber configuration. In order to increase the shear contribution, the span was reduced for the thickest specimens, and it led to an higher-than-expected scatter in the experimental results. It causes that from the perspective of material selection, it is not possible to draw a clear conclusion for the energy release rate. Besides, despite the scatter, glass fiber specimens still present a significant lower $\sigma_{\max } / \sigma_{\max _{V i r g i n}}$ ratio than the carbon specimens.

Further investigation would be needed with regards to the fiber treatment, a process commonly performed to improve the fibers adhesion with the surrounding resin. Fiber treatment could be tuned to ease the interaction with the nanofibers, improving bonding and the effectiveness of reinforce.

\section{FUnding ACKNOWLEDGEMENT STATEMENT}

This research received no specific grant from any funding agency in the public, commercial, or not-for-profit sectors

\section{REFERENCES}

[1] Y.A. Dzenis and D.H. Reneker. Delamination resistant composite prepared by small diameter fiber reinforcement at ply interfaces, 1999 .

[2] S. Ramakrishna. An introduction to electrospinning And nanofibers. World Scientific, 2005. 
[3] R. Palazzetti, A. Zucchelli, C. Gualandi, M.L. Focarete, L. Donati, G. Minak, and S. Ramakrishna. Influence of electrospun Nylon 6,6 nanofibrous mats on the interlaminar properties of Gr-epoxy composite laminates. Composite Structures, 94(2):571-579, January 2012.

[4] R. Panduranga, M.M. Sharpe, and K.N. Shivakumar. Assessment of polymer nanofiber interleaving in composite laminates through simple tests. In International SAMPE Technical Conference, 2012.

[5] K. Shivakumar and R. Panduranga. Interleaved polymer matrix composites - A review. In Collection of Technical Papers AIAA/ASME/ASCE/AHS/ASC Structures, Structural Dynamics and Materials Conference, 2013.

[6] S. Alessi, M. di Filippo, C. Dispenza, M.L. Focarete, C. Gualandi, R. Palazzetti, G. Pitarresi, and A. Zucchelli. Effects of Nylon 6,6 Nanofibrous Mats on Thermal Properties and Delamination Behavior of High Performance CFRP Laminates. 2014.

[7] A. Zucchelli, M.L. Focarete, C. Gualandi, and S. Ramakrishna. Electrospun nanofibers for enhancing structural performance of composite materials. Polymers for Advanced Technologies, 22(3):339-349, March 2011.

[8] J. Zhang, T. Yang, T. Lin, and C.H. Wang. Phase morphology of nanofibre interlayers: critical factor for toughening carbon/epoxy composites. Composite Science and Technology, 72(2):256-262, January 2011.

[9] I. Finegan. Modeling and characterization of damping in carbon nanofiber/polypropylene composites. Composites Science and Technology, 63(11):1629-1635, August 2003.

[10] X.F. Wu. Fracture of Advanced Polymer Composites with Nanofiber Reinforced interfaces. PhD thesis, University of Nebraska, 2003.

[11] K. Shivakumar, S. Lingaiah, H. Chen, P. Akangah, G. Swaminathan, and L. Russell. Polymer Nanofabric Interleaved Composite Laminates. AIAA Journal, 47(7):1723-1729, July 2009.

[12] P. Akangah, S. Lingaiah, and K. Shivakumar. Effect of Nylon- 66 nano-fiber interleaving on impact damage resistance of epoxy/carbon fiber composite laminates. Composite Structures, 92(6):1432-1439, May 2010.

[13] R. Palazzetti, X.T. Yan, and A. Zucchelli. Influence of geometrical features of electrospun Nylon 6,6 interleave on the CFRP laminates mechanical properties. Polymer Composites, 35(1):137-150, 2014.

[14] R. Palazzetti, A. Zucchelli, and I. Trendafilova. The self-reinforcing effect of nylon 6,6 nano-fibres on CFRP laminates subjected to low velocity impact. Composite Structures, 106:661-671, July 2013.

[15] F. Moroni, R. Palazzetti, A. Zucchelli, and A. Pirondi. A numerical investigation on the interlaminar strength of nanomodified composite interfaces. Composites Part B, 55:635-641, December 2013.

[16] H. Saghafi, A. Zucchelli, R. Palazzetti, and G. Minak. The effect of interleaved composite nanofibrous mats on delamination behavior of polymeric composite materials. Composite Structures, 109:41-47, March 2014.

[17] J. Yao, C. Bastiaansen, and T. Peijs. High Strength and High Modulus Electrospun Nanofibers. Fibers, 2:158-186, April 2014.

[18] ASTM D7264/D7264M. Standard test method for flexural properties of polymer matrix composite materials. Annual Book of ASTM Standards, 2007.

[19] European Structural Integrity Society; 1993. Protocol No 2 for interlaminar 
fracture toughness testing of composites: Mode II, 1993. 